\title{
A Solvent Free Synthetic Route for Cerium(IV) Metal-Organic Frame- works with UiO-66 Architecture and Their Photocatalytic Application
}

\author{
Matteo Campanelli, ${ }^{\#}$ Tiziana Del Giacco, ${ }^{\#,}$ Filippo De Angelis,,${ }^{\#,} \|_{\text {Edoardo Mosconi, }}{ }^{ \pm}$ \\ Marco Taddei,§ Fabio Marmottini, ,, Roberto D’Amato ${ }^{\#}$ and Ferdinando Costantino ${ }^{\#, \neq, *}$ \\ \#Dipartimento di Chimica, Biologia e Biotecnologie, Università di Perugia, Via Elce di Sotto 8, 06123 Perugia, Italy \\ ${ }^{\ddagger}$ Centro di Eccellenza Materiali Innovativi Nanostrutturati (CEMIN), Università di Perugia, Via Elce di Sotto 8, 06123 \\ Perugia, Italy \\ ${ }^{ \pm}$Computational Laboratory for Hybrid/Organic Photovoltaics (CLHYO), Istituto CNR di Scienze e Tecnologie Molecolari \\ (ISTM-CNR), Via Elce di Sotto 8, 06123 Perugia, Italy \\ "CompuNet, Istituto Italiano di Tecnologia, Via Morego 30, 16163 Genova, Italy \\ ${ }^{\S}$ Energy Safety Research Institute, Swansea University, Bay Campus, Fabian Way, Swansea, SA1, 8EN, UK
}

KEYWORDS :Metal-Organic Frameworks, Cerium, Photocatalysis, DFT Calculations, Green Syntheses.

\begin{abstract}
A near solvent-free synthetic route for Ce-UiO-66 metal-organic frameworks (MOFs) is presented. The MOFs are obtained by simply grinding the reagents, cerium ammonium nitrate (CAN) and the carboxylic linkers, in a mortar for few minutes with the addition of a small amount of acetic acid $(\mathrm{AcOH})$ as modulator $(8.75 \mathrm{eq}, 0.5 \mathrm{~mL})$. The slurry is then transferred into a $2 \mathrm{~mL}$ vial and heated at $120^{\circ} \mathrm{C}$ for 1 day. The MOFs have been characterized for their composition, crystallinity and porosity and employed as heterogeneous catalysts for the photo-oxidation reaction of substituted benzylic alcohols to benzaldaldehydes under near ultraviolet light irradiation. The catalytic performances, such as selectivity, conversion and kinetics, exceed those of similar systems studied by chemical oxidation using similar Ce-MOFs as catalyst. Moreover, the MOFs were found to be reusable up to three cycles without loss of activity. Density functional theory (DFT) calculations were used in order to fully describe the electronic structure of the best performing MOFs and to provide useful information on the catalytic activity experimentally observed.
\end{abstract}

\section{Introduction}

Metal-organic frameworks (MOFs) are a class of porous crystalline materials constituted of metallic clusters and organic linkers connected in an ordered fashion and containing accessible channels to gases and solvents. They have attracted increasing attention during the past two decades due to their high porosity, stability, tunable structures and controllable surface functionalities. Therefore, in view of potential industrial application, increasing efforts are being made to develop large scale and sustainable MOFs syntheses .${ }^{1-6}$ However, most MOFs are still in an early development stage and have not yet been brought into the market place. In 2008 , the catalysis section at University of Oslo published the synthesis of UiO-66, the first zirconium-based MOF (Zr-MOF) reported, built from $\mathrm{Zr}_{6} \mathrm{O}_{4}(\mathrm{OH})_{4}{ }^{12+}$ nodes and 1,4-benzene-dicarboxylate (BDC) linkers, which exhibits exceptional chemical and thermal stability compared to other known MOFs. ${ }^{7}$ Furthermore, the UiO-66 series can be tuned by partial/total linker or node functionalization. ${ }^{8-10}$ A large number of Zr-MOFs based on different clusters and with variable structure and porosity has been recently reported. ${ }^{11-13}$ More recently, the chemistry of $\mathrm{Ce}(\mathrm{IV})$ based MOFs has rapidly grown because the use of Ce(IV) allows to get MOFs isostructural to Zrhomologues with remarkable stability and with potential interest in the field of redox and photo-catalysis. Stock and co-workers first reported in 2015 the $\mathrm{N}, \mathrm{N}$-dimethylformamide (DMF) based synthesis of Ce-UiO-66 MOFs with several dicarboxylic ligands demonstrating the strong homologies between the $\mathrm{Zr}$ and $\mathrm{Ce}$ based hexanuclear clusters. ${ }^{14}$ Other topologies, based on the same building units were reported later on. ${ }^{15-17}$ Finally, the syntheses of mixed Zr-Ce MOFs and water based syntheses of Ce-MOF were recently presented. ${ }^{18,19}$

Photocatalytic applications of MOFs have also been widely explored due to their semiconductor properties. ${ }^{20,21}$ When a semiconductor is irradiated with energy equal to or greater than their band gap, charge separated states are generated, with the electrons on the valence band (VB) excited to the conduction band (CB), leaving positive holes $\left(\mathrm{h}^{+}\right)$in the VB. These photogenerated charge carriers (electrons and holes) migrate separately to the surface of the semiconductor particles and react with surface-adsorbed substrates to promote photocatalytic reactions. ${ }^{22}$ The rational design and modification of MOFs structures enables control on the chemical and 
physical properties of photocatalysts at molecular level. So far, Silva and co-workers firstly synthesized UiO-66-NH $\mathrm{N}_{2}$ by replacing $\mathrm{H}_{2} \mathrm{BDC}$ by $2-\mathrm{NH}_{2}$-terephthalic acid in the synthesis ${ }^{23}$ and $\mathrm{Wu}$ and co-workers studied the photocatalytic selective oxidation of alcohols to their corresponding aldehydes and reduction of aqueous $\mathrm{Cr}$ (VI) utilizing this MOF as the photocatalyst. ${ }^{24}$ Following this research direction, a series of mixed-linker Zr-based MOFs have been synthesized in one pot reactions for the visible-light photocatalytic oxidation of alcohol by Goh and co-workers. ${ }^{25}$

The research on novel efficient photocatalysts focussed our attention on cerium based MOFs. A recent paper of Truhlar and coauthors reported the application of Ce-MOF in photocatalysis from a theoretical point of view. ${ }^{26}$ In light of this, its forecast encourages the synthesis and experimental application in several reactions driven by light. The exchange of metal center of MOFs does not show any effect on the photocatalytic activity. ${ }^{22}$ In Ce-MOFs, ligand-to-metal charge transfer (LMCT) can bring about the separation of photogenerated charges, promoted by the low-lying empty $4 \mathrm{f}$ orbitals of $\mathrm{Ce}^{4+}$. Another important structural variation that can be used to design a promising UiO-66(Ce) photocatalyst, is linker functionalization. ${ }^{26}$ Combining these ideas, herein we present a new green, solvent free, synthetic methodology to get crystalline Ce-MOF with several substituted dicarboxylic ligands, as well as the electronic structure obtained by DFT calculations and the application in photo-oxidation reaction of substituted benzyl alcohols to aldehydes.

\section{Results and discussion}

\section{Synthesis ad characterization}

The linkers used for the synthesis of Ce-MOFs are shown in Scheme 1. The corresponding MOFs are named as Ce-UiO-66-X (with $\mathrm{X}=\mathrm{H}, \mathrm{Br}, \mathrm{NO} 2)$, Ce-UiO-66-4F, Ce-MIL140A-4F and Ce-UiO-66-PDC.<smiles>O=C(O)c1ccc(C(=O)O)cc1</smiles>

H-BDC<smiles>O=C(O)c1ccc(Br)c(Br)c1</smiles>

$\mathrm{Br}-\mathrm{BDC}$

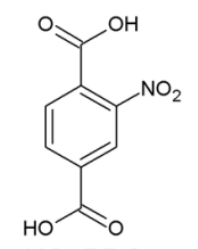

$\mathrm{NO}_{2}-\mathrm{BDC}$

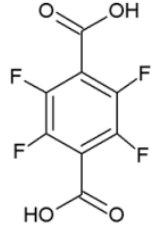

F4-BDC

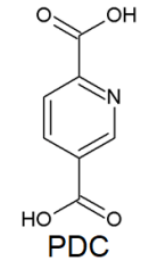

PDC

Scheme 1. Molecular structure of the linkers used.

A near solvent free strategy is here proposed to obtain Ce-UiO-66. The first step consists in an energetic grinding of the metal salts and linkers with a small amount of acetic acid $(0.5 \mathrm{~mL}, 8.75 \mathrm{eq})$, followed by heating at temperatures comprised in the $110-120{ }^{\circ} \mathrm{C}$ range in small vials $(1 \mathrm{~mL})$ in a custom-made aluminium reactor block. Syntheses performed with larger volume vials generally lead to products with low crystallinity. The method here presented is similar to a Liquid-Assisted Grinding (LAG) being the ratio liquid/solid reagents comprised in the $0.1-1$ range, according to recent definitions in the field of solvent-free syntheses of MOFs. ${ }^{27}$ This kind of procedures was also recently used for the syntheses of several $\mathrm{Zr}-\mathrm{MOFs}{ }^{28-32}$ However, in the present work the solids are also kept at high temperature in closed vials after grinding under acetic acid vapour pressure, in a way similar to that called accelerated aging. ${ }^{27}$ The synthesis here reported uses a low amount of acetic acid (8.75 eq. respect to Ce) to obtain the products, unlike recently reported works. ${ }^{33,34}$ Besides, to the best of our knowledge, Ce-UiO-66-Br was obtained here for the first time. X-Ray Powder Diffraction (XRPD) patterns (Figure 1) show the typical face-centered cubic UiO-66 fcu phase peak positions at about $7.1^{\circ}$ and $8.2^{\circ}$ of $2 \theta$, which correspond to the lattice planes (111) and (002), respectively. In addition to the UiO-66 phases the use of F4-BDC also afforded the F4_MIL-140A(Ce) phase which was recently reported via other synthetic procedures. ${ }^{17}$

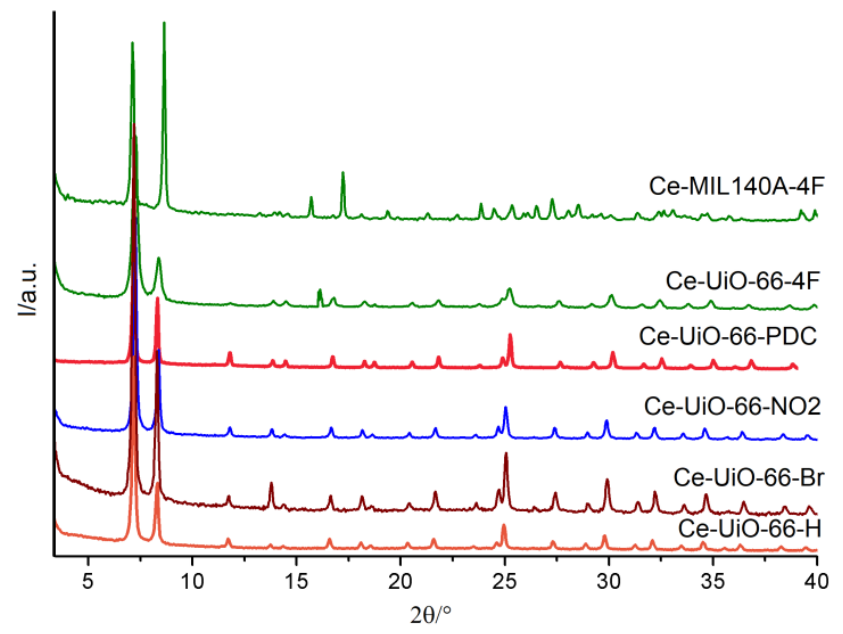

Figure 1. XRPD patterns of the Ce-MOFs. 
The $\mathrm{N}_{2}$ adsorption-desorption isotherms at $77 \mathrm{~K}$ obtained with the Ce-MOF samples are shown in Figure 2. From the Brunauer Emmett Teller (B.E.T.) and t-plot analyses of the adsorption data, the specific surface area and micropore volumes were calculated and reported in Table S1. The micropore volume values indicate that all the samples are microporous. In the case of Ce-UiO-66-H and Ce-UiO-66-PDC the micropore volumes were found to be 20 and 35\% smaller than the values of analogous MOFs reported in the literature, whereas for the MOF with the tetrafluoroterephthalate linker, they match quite well with the analogous materials obtained by water-based syntheses. ${ }^{14,17,35}$ This could be likely due to the formation of a certain percentage of non-porous impurities such as cerium oxide, although the XRPD patterns show only the peaks belonging to the MOF structure.

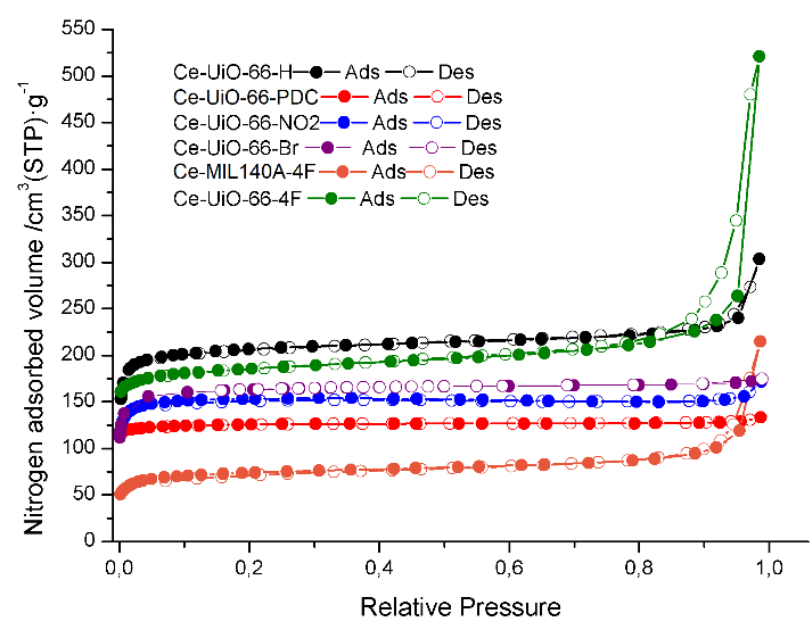

Figure 2. N2 adsorption isotherms for Ce-MOFs.

Figure 3 displays the SEM images of the synthesized MOFs. They have irregular octahedral shape with size ranging between 30 and $150 \mathrm{~nm}$, as reported in previous studies. ${ }^{14,17,35}$

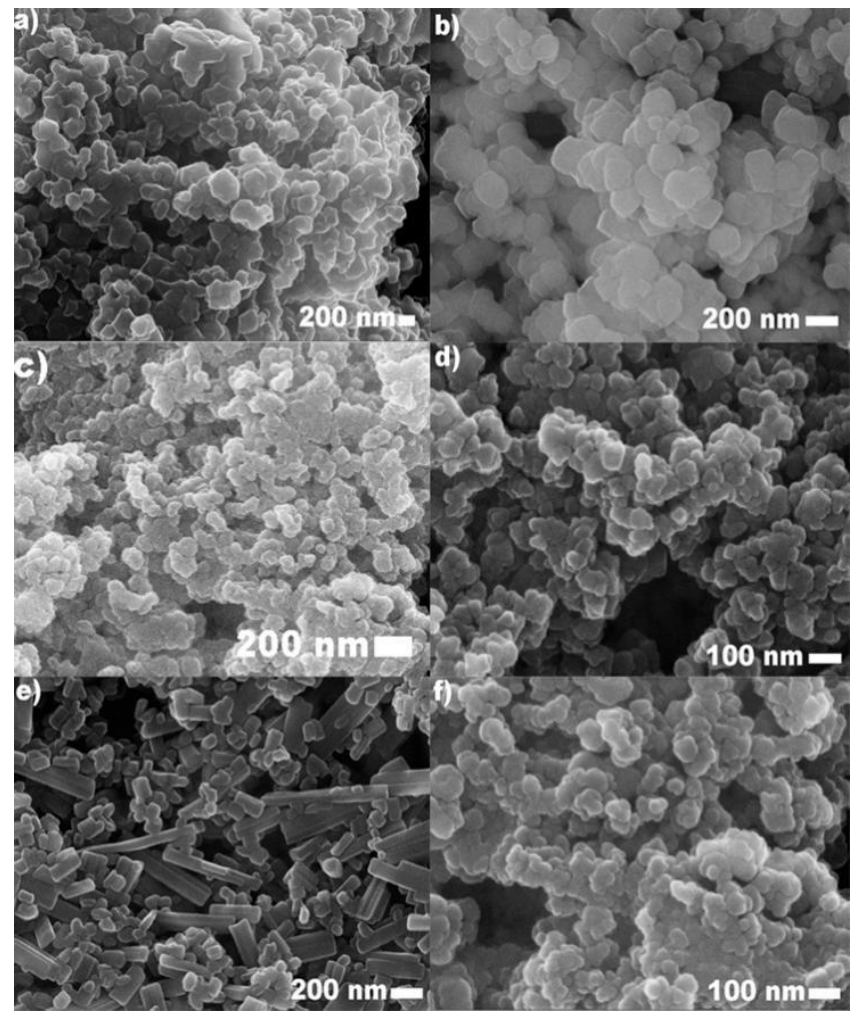

Figure 3. FE-SEM images of Ce-MOFs: a) Ce-UiO66-H; b) Ce-UiO66-Br; c) Ce-UiO66-NO2; d)Ce-UiO66-4F ; e) Ce-MIL140A4F; f) Ce-UiO-66-PDC. 
The morphology of the Ce-MIL140A-4F MOF, here observed for the first time, is rod-like as in the case of the isoreticular analogue of $\mathrm{Zr}^{36}$ Focusing on this image, UiO impurities are, very likely, present in the sample as small octahedral crystals (Figure 3 , e). Regarding the stability, TGA analysis (Figure S1) shows that the weight loss of all Ce-MOFs is the same to those already reported. ${ }^{14,17,35}$ However, Ce-UiO66-H and Ce-UiO66-Br are more stable than the other functionalized MOFs. It is known that CeUiO66-4F undergoes explosive decomposition, which leads to ejection of powder from the sample holder, as evidenced by the vertical loss in the TGA curve of this MOF (Figure S2). ${ }^{17}$ The same behaviour was also observed for Ce-UiO-66-NO2 sample which exhibited a vertical drop of mass at T higher than $250{ }^{\circ} \mathrm{C}$ (Figure S2). In order to detect the presence of impurities or unreacted reagents, ionic chromatography (IC) was also performed on digested samples. The presence of nitrates was detected only in the case of Ce-UiO-66PDC sample $(1.5 \mathrm{mmol} / \mathrm{g})$. Very likely, the nitrate ion is present as counter anion of the protonated pyridine group. FT-IR spectra are reported in Figure S2. ${ }^{1} \mathrm{H}-\mathrm{NMR}$ spectra (Figure S3) were also recorded on digested samples in order to check the presence of acetic acid as monocarboxylic substituent. Notably, no AcOH was detected in all samples suggesting that the MOFs could be defect free.

Table 1 reports the $\lambda_{\max }$ and $\mathrm{E}_{\max }$ values of the solid state UV-Vis absorption spectra of Ce-MOFs. The spectra (Figure S4) show a single broad absorbance band with the maximum in the near Vis region. The corresponding $\mathrm{E}_{\text {onset }}$ provides the estimated Band-Gap values for all the samples.

Table 1. $\lambda_{\max }$, and $\mathbf{E}_{\max }$ obtained from solid state absorbance measurements.

\begin{tabular}{|l|l|l|l|l|}
\hline \multicolumn{1}{|c|}{ Sample } & \multicolumn{1}{|c|}{$\begin{array}{c}\lambda_{\text {onset }} \\
(\mathrm{nm})\end{array}$} & $\begin{array}{c}\mathrm{E}_{\text {onset }} \\
(\mathrm{eV})\end{array}$ & $\begin{array}{c}\lambda_{\max } \\
(\mathrm{nm})\end{array}$ & \multicolumn{1}{|c|}{$\begin{array}{c}\mathrm{E}_{\max } \\
(\mathrm{eV})\end{array}$} \\
\hline $\mathrm{Ce}-U i O 66-\mathrm{H}$ & 434 & 2.86 & 301 & 4,12 \\
\hline $\mathrm{Ce}-U i O 66-\mathrm{Br}$ & 457 & 2.71 & 316 & 3,92 \\
\hline $\mathrm{Ce}-U i O 66-\mathrm{NO}_{2}$ & 420 & 2.95 & 320 & 3,87 \\
\hline Ce-UiO66-4F & 458 & 2.71 & 300 & 4,13 \\
\hline Ce-MIL140A-4F & 448 & 2.77 & 305 & 4,06 \\
\hline Ce-UiO-66-PDC & 460 & 2.70 & 324 & 3,82 \\
\hline
\end{tabular}

\section{Photocatalytic tests}

All MOFs were activated at $120{ }^{\circ} \mathrm{C}$ overnight before use. This treatment was used in order to remove water molecules on the catalyst pore surface, which can compete with the adsorption of the substrate. At first, in order to test the catalytic efficacy, the oxidation of 4-methoxybenzyl alcohol (MeOBA) by Ce-UiO66-H (pristine) was chosen as a probe reaction. We selected this alcohol because its redox potential is lower than that of other benzyl alcohol derivatives. The oxidation of $\mathrm{MeOBA}$ in ethanol (EtOH) as solvent, photocatalyzed by Ce-UiO66-H and carried out under $355 \mathrm{~nm}$ light irradiation (wavelength absorbed only by the catalyst), produced the corresponding benzaldehyde (Scheme 2) in quantitative yield within 90 min. Ethanol proved to be an optimal solvent because it is polar enough both to dissolve the substrate and to stabilize the ionic intermediates known to form in the photo-oxidation reaction (see below). The use of this solvent was possible because it was inert to oxidation; in fact no acetaldehyde trace was found among the products (see Figure S8). We also carried out the photocatalysis in $\mathrm{H}_{2} \mathrm{O}$ and MeCN, but EtOH was the solvent in which the oxidation of 4-methoxybenzyl alcohol was more efficient (see Figure S9).<smiles>COc1ccc(CO)cc1</smiles>

Scheme 2. Photo-oxodation of MeOBA to 4-methoxy benzaldehyde.

Oxidation experiments performed in the dark, or in the absence of $\mathrm{O}_{2}$ (under Ar), or with CAN (a potential strong oxidant under irradiation $)^{37}$ instead of MOF, did not yield any product in all cases. The second step was to examine the substituent effect, such as $\mathrm{Br}, \mathrm{NO}_{2}$ and $\mathrm{F}$, of the BDC linker on the photo-oxidation yield of MeOBA. As observed from the data reported in Table 2 and compared to those of the pristine MOF, in the case of Ce-UiO66-NO2 a significant lower conversion was observed, obtaining quantitatively the aldehyde after $150 \mathrm{~min}$. Ce-UiO66-4F was even less reactive, producing only $2 \%$ of oxidation product after 90 min. No aldehyde was detected when the MIL-140_A analogue was employed. A possible explanation for this behavior can be that the small pores of both F4-BDC derivatives do not allow an efficient diffusion of alcohols molecules inside. A slightly higher yield than that 
of pristine MOF was achieved with $\mathrm{Ce}-\mathrm{UiO}-66-\mathrm{Br}$, which reached the maximum value of product yield within 80 min. From these results it is evident that the weak electron-withdrawing substituents favor the photocatalysis. Anyway, the highest reactivity was shown by the Ce-UiO-66-PDC MOF. Indeed, aldehyde complete conversion was obtained just after 75 min, without any evidence of the corresponding acid (see Figure S6). High selectivity was observed in all reactions, contrary to what reported by various previous studies, ${ }^{38-41}$ reporting that the photocatalysis of aromatic alcohols is often a not selective process. ${ }^{42}$

Table 2. Photo-oxidation of MeOBA. ${ }^{\mathrm{a}}$

\begin{tabular}{|l|l|l|l|}
\hline \multicolumn{1}{|c|}{ Photocatalyst } & \multicolumn{1}{|c|}{$\begin{array}{c}\mathrm{t}_{\text {irr }} \\
(\mathrm{min})\end{array}$} & $\begin{array}{c}\text { Alcohol }^{\mathrm{b}} \\
(\%)\end{array}$ & \multicolumn{1}{|c|}{$\begin{array}{c}\text { Aldehyde }^{\mathrm{b}} \\
(\%)\end{array}$} \\
\hline CAN & 60 & 90 & 0 \\
\hline Ce-UiO-66-H & 90 & 94 & 0 \\
\hline Ce-UiO66-H & 90 & 0 & 95 \\
\hline Ce-UiO66-Br & 80 & 0 & 94 \\
\hline Ce-UiO66-NO2 & 150 & 0 & 94 \\
\hline Ce-UiO66-4F & 90 & 93 & 2 \\
\hline Ce-MIL140A-4F & 60 & 95 & 0 \\
\hline Ce-UiO-66-PDC & 75 & 0 & 94 \\
\hline
\end{tabular}

${ }^{\mathrm{a}}[\mathrm{MeOBA}]=1.00 \times 10-2 \mathrm{M},[$ Photocatalyst $]=8.25 \times 10^{-4} \mathrm{M}$, under Oxygen flux, with $\lambda=355 \pm 20 \mathrm{~nm}$. ${ }^{\mathrm{b}}$ Yields refer to the initial amounts of substrate. The error is $\pm 5 \%$. ${ }^{c}$ Experiment under Argon atmosphere.

Moreover, in our case the reaction times are in general rather short compared to other examples of photocatalyzed oxidations by MOFs already reported. ${ }^{24}$ All these results were rationalized with the mechanism schematized in Scheme 3 , which was formulated on the basis of that already known for the heterogeneous photocatalysis with titanium dioxide. ${ }^{42}$
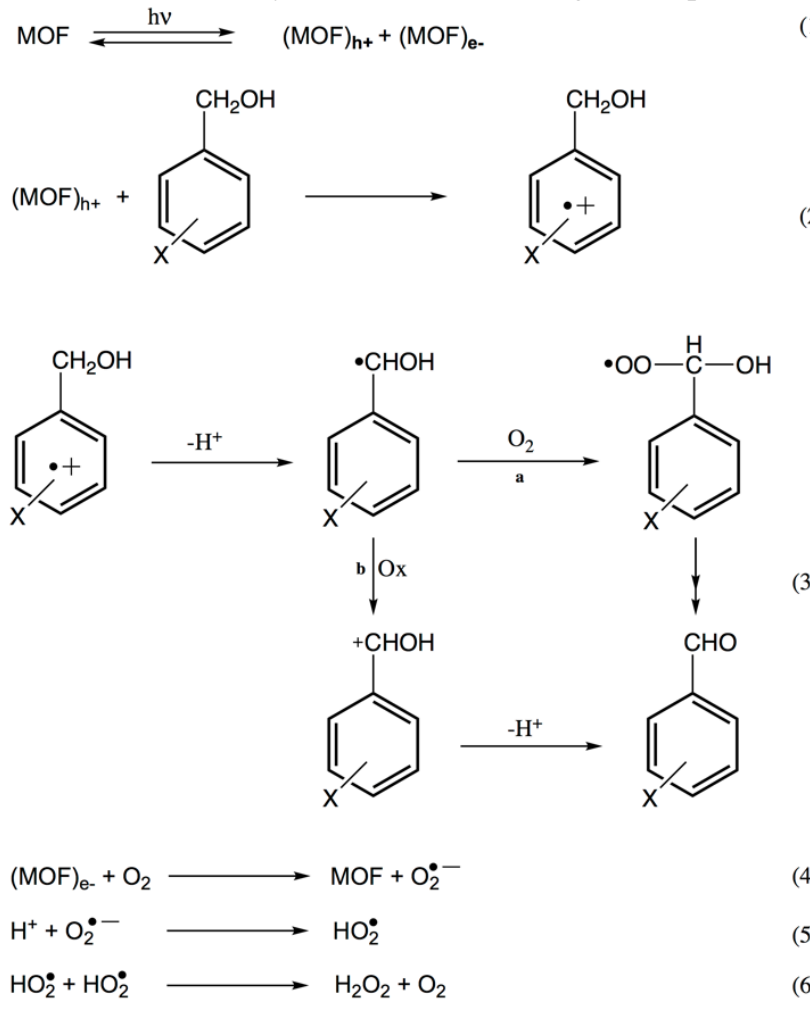

(1)

(3)

Scheme 3. Mechanism of photo-oxidation of benzylic alcohols with MOFs.

The first stage involves the absorption of light by the MOF, in particular by the linker, with consequent charge separation involving the electron located on the metal and the positive hole on the linker. ${ }^{20,22,23}$ The alcohol forms the corresponding radical cation by electron transfer from the aromatic ring to the positive hole (eq. 2); this intermediate, being more acidic than the corresponding neutral 
substrate, deprotonates to 4-methoxy- $\alpha$-hydroxy benzyl radical (eq. 3), which evolves, by reaction with molecular oxygen, to the final oxidation product (eq 3, path a). ${ }^{42,43}$ Another plausible reaction path of the radical could be its oxidation by MOF to the corresponding 4-methoxy- $\alpha$-hydroxy benzyl cation, which leads to the formation of aldehyde by subsequent proton loss. For as concerns the reduced metal, a back electron transfer with $\mathrm{O}_{2}$ is hypothesized, which regenerates the MOF in its initial oxidation state, together with the superoxide anion (eq. 4), which then evolves to hydrogen peroxide and oxygen in the presence of acid (eqq. 5 and 6 ). Indeed, hydrogen peroxide was recovered in the reaction mixture, as described in Supporting Information (Figure S7). Obviously, the reaction efficiency of the hole with alcohol will be conditioned by the rate of its recombination with the electron to reform the initial MOF (back reaction of eq. 1).

The experimental data point out that the reactivity is exactly the opposite of the theoretical oxidative power $\left(\mathrm{BDC}^{-\mathrm{NO}} \mathrm{B}_{2}>\mathrm{BDC}-\mathrm{H}>\right.$ BDC-Br), expressed by their vacuum aligned HOCO (highest occupied crystal orbitals) energy, equal to $-7.57,-6.73$ and -6.19 eV, respectively. ${ }^{25}$ The reason of this trend is rather to be sought on the effects that influence the lifetime of the oxidizing hole. It is known that, when Ce-MOFs are excited, the photogenerated electrons undergo LMCT to form charge-separated states, so preventing the rapid recombination of the photogenerated charges. ${ }^{26}$ The kinetics of this process can be related with the LMCT energy, $\mathrm{E}_{\mathrm{LMCT}}$, which is defined as the energy change upon transferring the photogenerated electron from the photoexcited linker orbital to the lowest unoccupied metal orbital. This means that the substituents on the linker not only influence the oxidative ability of MOF, but also the efficiency of separation of the photogenerated charges. In particular, $\mathrm{E}_{\mathrm{LMCT}}$ will be all the more negative, i.e. the electron-hole separation will be the more favored, as the substituent is less electron-withdrawing. Accordingly, also the electron-hole recombination rate, i.e. the competitive reaction with the photo-oxidation, will slow down. $\mathrm{E}_{\mathrm{LMCT}}$ values for Ce-BDC-Br, Ce-BDC-H and Ce-BDC$\mathrm{NO}_{2}$ are $-1.42,-1.43$ and $-1.33 \mathrm{eV}$, respectively, ${ }^{25}$ which are perfectly in line with the similar reactivity of the first two catalysts and with the lower reactivity of the third one. In the case of Ce-UiO-66-4F, the presence of four electron-withdrawing substituents as fluorine on the linker ring could increase the $\mathrm{E}_{\mathrm{LMCT}}$ value, favoring further the electron-hole recombination and this is in agreement with the lowest reactivity observed for this MOF. ${ }^{26}$ Use of pyridine-2,5 dicarboxylates as a linker produces a MOF (Ce-UiO-66-PDC) able to convert quantitatively MeOBA to the corresponding aldehyde in $75 \mathrm{~min}$, a time shorter than that with Ce-BDC-Br. Reasonably, the electron-withdrawing effect of nitrogen of the pyridine could be similar to that of a bromide on a benzene ring, but a contribution due to the HOCO energy cannot be excluded.

With the aim of providing more quantitative information on the reactivity, a systematic study was performed on the photo-oxidation of a series of benzyl alcohol derivatives (benzyl alcohol, BA; 4-trifluoromethylbenzyl alcohol, CF3BA; 4-chlorobenzyl alcohol, ClBA) by the best performing MOF, i.e. Ce-UiO-66-PDC. Interestingly, the only product obtained from the Ce-UiO-66-PDC photocatalyzed oxidation of each alcohol, in oxygenated EtOH, was the corresponding benzaldehyde (Table S3), as already observed for MeOBA, proving that the process is always highly selective. All alcohols investigated were found to be less reactive than MeOBA. In fact, while the complete conversion of MeOBA occurred within $75 \mathrm{~min}$, that of BA was quantitative within 105 min. Increasing the electron-withdrawing ability of the substituent, i.e. going from $\mathrm{Cl}$ to $\mathrm{CF}_{3}$, the reactivity further decreased: after $360 \mathrm{~min}, 87 \%$ of 4-chlorobenzaldehyde and only $19 \%$ of 4-trifluoromethylbenzaldehyde were formed. Thus, the reactivity decreases with the increase of the oxidation potential, which extends the range from $1.5 \mathrm{~V}$ for MeOBA to $2.7 \mathrm{~V}$ for CF3BA (oxidation potentials determined in $\mathrm{MeCN}$ vs. SCE) ${ }^{41}$ These evidences lead to suppose that, on the basis of the previously discussed photo-oxidation mechanism (Scheme 3), the electron transfer step (eq. 2) is certainly rate determining. In addition, the reactivity of MeOBA with Ce-UiO-66-PDC was identical to that of the corresponding deuterated alcohol on the benzylic position (4- $\left.\mathrm{OCH}_{3}-\mathrm{C}_{6} \mathrm{H}_{4} \mathrm{CD}_{2} \mathrm{OH}\right)(\mathrm{Table} \mathrm{S} 3)$. The lack of kinetic isotopic effect of deuterium leads us to conclude that the slowest step is not the radical cation deprotonation (eq. 3), but the electron transfer step. It is interesting to compare our results with those obtained by Shen et al, ${ }^{24}$ who carried out the photo-oxidation of some benzylic alcohols catalyzed by $\mathrm{Zr}-\mathrm{NH}_{2}-\mathrm{UiO}-66$. Firstly, the $\mathrm{Zr}-\mathrm{NH}_{2}-\mathrm{UiO}-66$ system showed much lower selectivity for the aldehyde with electron withdrawing substituted alcohols compared with that obtained with our MOFs. Furthermore, the reactivity of the benzyl alcohols increased with the electron withdrawing ability of the substituent and thus the deprotonation reaction at the benzylic position as rate-determining step was hypothesized. The different behavior may be due to the lower reactivity of our catalyst compared to that of Shen. Anyway, to rationalize the diverse oxidative reactivity of the two systems is complex, considering the many factors involved in heterogeneous photocatalysis.

Recycling attempts, up to three times, were carried out by using Ce-UiO-66-PDC in the photo-oxidation reaction of MeOBA. After each reaction, the catalyst was separated from the reaction mixture by filtration, washed with $\mathrm{CH}_{2} \mathrm{Cl}_{2}$, heated at $100{ }^{\circ} \mathrm{C}$ for $1 \mathrm{~h}$ in order to remove $\mathrm{CH} 2 \mathrm{Cl} 2$, then washed again with $\mathrm{H}_{2} \mathrm{O}$ and, finally, dried under vacuum at room temperature. For each cycle, $\mathrm{X}$-ray diffraction patterns were collected (Figure S5). The photocatalyst showed to be successfully recyclable; indeed, after 75 min, the aldehyde yield was of $98 \%$ after each photo-oxidation. On the basis of these results, we can state that the MOF is subject to low leaching phenomena, covering of the active phase or sintering, despite not having been subjected to any treatment between one cycle and another. Release of Ce in solution was investigated using Inductively Coupled Plasma Optical Emission Spectroscopy (ICP-OES). The results reported in Table S4 show that Ce leaching after three photocatalytic experiments is very low $(<1 \%)$.

\section{Theoretical calculations}

To gain insight into the electronic and optical properties of these materials, DFT simulations have been carried out to evaluate the density of states (DOS) of Ce-UiO-66-H and Ce-UiO-66-PDC. Following a similar approach proposed by Truhlar and coworkers, ${ }^{26}$ 
the electronic properties were simulated with the HSE06 hybrid functional as implemented in VASP program package ${ }^{44,45}$ (see Supporting Information for theoretical details). As already reported HSE06 nicely reproduces band gaps of various functionalized UiO$66(\mathrm{Zr})$ showing quantitative agreement with the experimental values. ${ }^{46}$ For Ce-UiO-66-H, we calculated a band gap of 2.99 while for Ce-UiO-66-PDC we obtain a value of $2.79 \mathrm{eV}$, in excellent agreement with the absorption onset ( $\lambda_{\text {onset }}$ ) reported in Table 1 . As we can see from the DOS analysis in Figure 4, the organic molecules mainly contribute to valence band (VB) states while the CB is mainly constituted by the Ce states. To compare the relative VB and CB energy levels, the Ce-UiO-66-H and Ce-UiO-66-PDC DOS was aligned to the $\mathrm{Ce}$ signal present at around $-15 \mathrm{eV}$ and were set to zero at the Ce-UiO-66-H HOMO level. Interestingly, we found that the decrease of the band gap found for Ce-UiO-66-PDC is essentially related to an upshift of $\mathrm{VB}$ of about $0.2 \mathrm{eV}$ due to the presence of an additional band associated to the to the pyridine states (see in Figure $4 \mathrm{~b}$ the $\mathrm{N}$ contribution in blue). In particular, while for $\mathrm{Ce}-\mathrm{UiO}-66-\mathrm{H}$ we found one main intense band at the VB edge, for Ce-UiO-66-PDC we observe two different bands: one at around $-0.1 \mathrm{eV}$ characterized by the $\mathrm{C}$ and $\mathrm{O}$ contribution and one at $0.2 \mathrm{eV}$ showing the contribution of $\mathrm{N}, \mathrm{O}$ and $\mathrm{C}$. As a matter of fact, in the Ce-UiO-66-PDC system the pyridine moiety introduces an additional occupied band that is responsible of the upshift of the VB leading to the decreasing of the band gap.
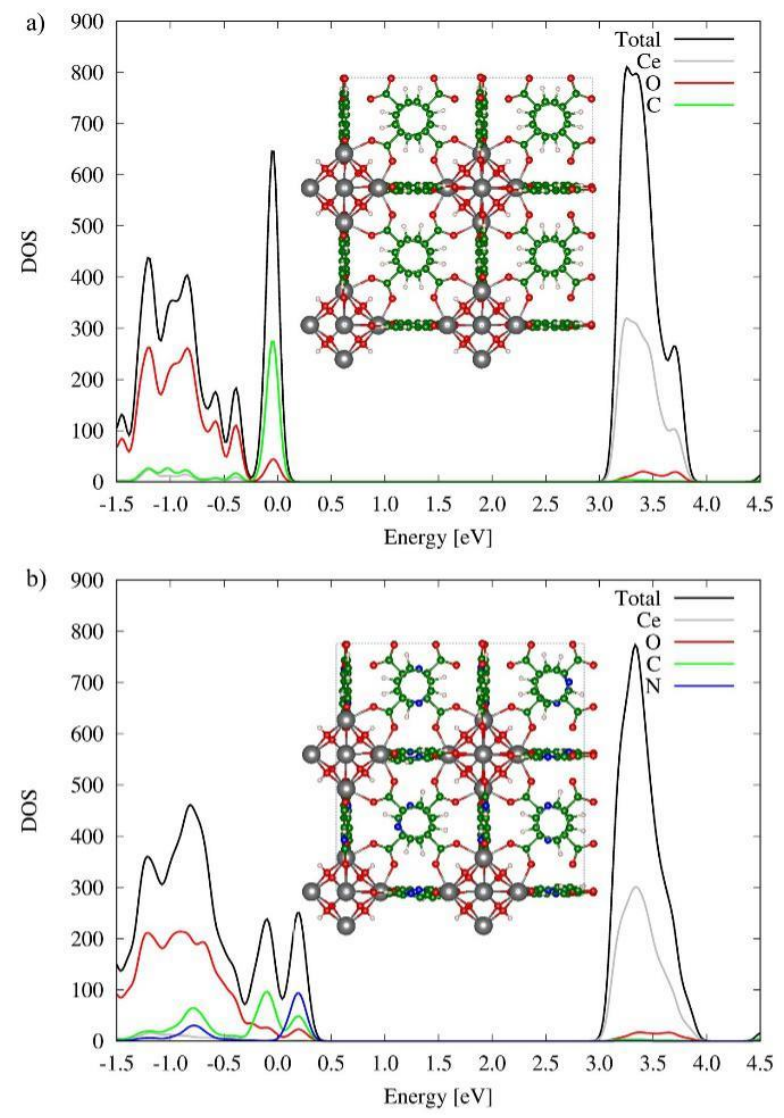

Figure 4. DOS analysis of (a) Ce-UiO-66-H and (b) Ce-UiO-66-PDC in black. Separated contribution of the atomic species to the DOS are also reported in gray $(\mathrm{Ce})$, red $(\mathrm{O})$, green $(\mathrm{C})$ and blue $(\mathrm{N})$.

\section{Conclusions}

In summary, a new quick and easy synthetic solvent-free methodology for obtaining Ce-UiO-66 MOFs is here presented. The procedure makes use of a very small amount of acetic acid as modulator and no further solvents are needed. The MOFs were obtained by using different substituted BDC linkers and 2,5-pyridinedicarboxylic acid. Their optical properties were investigated both from the theoretical and the experimental point of view. The effect of band-gap shift of the materials upon near UV light is due to the molecular structure of the linker which induced different LMCT. The MOFs display good photocatalytic properties towards the photooxidation of substituted benzylic alcohols to aldehydes. The activity of the photocatalytic systems depends on the electron withdrawing effects of the aromatic substituent. Further investigation will be devoted to fine tuning the band gap of the Ce-MOFs by choosing appropriate linkers for other key reactions to be tested upon photoirradiation. 


\section{ASSOCIATED CONTENT}

Supporting Information available. Synthetic and analytical procedures, experimental and instrumental details, TGA curves, UV-Vis, FTIR and ${ }^{1} \mathrm{H}-\mathrm{NMR}$ spectra, additional catalytic data, additional XRPD patterns, theoretical calculations details. This material is available free of charge via the Internet at http://pubs.acs.org.

\section{AUTHOR INFORMATION}

\section{Corresponding Author}

*ferdinando.costantino@unipg.it.

\section{PresentAddresses}

$\dagger$ If an author's address is different than the one given in the affiliation line, this information may be included here.

\section{Author Contributions}

The manuscript was written through contributions of all authors. / All authors have given approval to the final version of the manuscript. /

\section{ACKNOWLEDGMENT}

The present work was supported by Fondo Ricerca di Base (FRB-2017) and the Project AMIS, Department of Excellence University of Perugia. M.T. acknowledge the European Union's Horizon 2020 research and innovation programme under the Marie Skłodowska-Curie grant agreement No 663830 .

\section{REFERENCES}

(1) Silva, P.; Vilela, S. M. F.; Tomè, J. P. C.; Almeida Paz, F. A. Multifunctional Metal-Organic Frameworks: From Academia to Industrial Applications. Chem. Soc. Rev. 2015, 44, 6774-6803.

(2) Farha, O. K.; Yazaydin, A. O.; Eryazici, I.; Malliakas, C. D.; Hauser, B. G.; Kanatzidis, M. G.; Nguyen, S. T.; Snurr, R. Q; Hupp, J. T. De Novo Synthesis of a Metal-Organic Framework Material Featuring Ultrahigh Surface Area and Gas Storage Capacities. Nat. Chem. 2010, 2, 944-948.

(3) Farha, O. K; Eryazici, I; Jeong, N. C; Hauser, B.G; Wilmer, C. E; Sarjeant, A. A; Snurr, R. Q; Nguyen, S. T; Yazaydin, A. O; Hupp, J. T. Metal-Organic Framework Materials with Ultrahigh Surface Areas: Is the Sky the Limit? J. Am. Chem. Soc. 2012, 134, 15016-15021.

(4) Eddaoudi, M.; Kim, J; Rosi, N.; Vodak, D.; Wachter, J; O’Keeffe, M.; Yaghi, O.M. Systematic Design of Pore Size and Functionality in Isoreticular MOFs and Their Application in Methane Storage. Science 2002, 295, 469-462

(5) Kim, J.; Yazaydin, A. O.; Furukawa, H.; Choi, E.; Snurr, R. Q.; Choi, S. B.; Go, Y. B.; Yaghi, O. M.; Aratani, N.; Ko, N. Ultrahigh Porosity in Metal-Organic Frameworks. Science 2010, 329, 424-428.

(6) Stock, N.; Biswas, S. Synthesis of Metal-Organic Frameworks (MOFs): Routes to Various MOF Topologies, Morphologies, and Composites. Chem. Rev.2012, 112, 933-969.

(7) Cavka, J.H.; Jakobsen, S.; Olsbye, U.; Guillou, N.; Lamberti, C.; Bordiga, S.; Lillerud, K.P.A New Zirconium Inorganic Building Brick Forming Metal Organic Frameworks with Exceptional Stability. J. Am. Chem. Soc. 2008, 130, 13850-13851.

(8) Kandiah, M.; Nilsen, M. H.; Usseglio, S.; Jakobsen, S.; Olsbye, U.; Tilset, M.; Larabi, C.; Quadrelli, E. A.; Bonino, F.; Lillerud, K. P. Synthesis and Stability of Tagged UiO-66 Zr-MOFs. Chem. Mater. 2010, 22, 24, 6632-6640.

(9) Lee, Y.; Kim, S.; Kang, J. K.; Cohen, S. M. Photocatalytic $\mathrm{CO}_{2}$ reduction by a mixed metal (Zr/Ti), mixed ligand metal-organic framework under visible light irradiation. Chem. Commun. 2015, 51, 5735-5738.

(10) Abednatanzi, S.; Gohari Derakhshandeh, P.; Depauw, H.; Coudert, F.-X.; Vrielinck, H.; Van Der Voort, P.; Leus, K. Mixed-Metal MetalOrganic Frameworks. Chem. Soc. Rev. 2019, 48, 2535-2565.

(11) Mondloch, J. E.; Bury, W.; Fairen-Jimenez, D.; Kwon, S.; Demarco, E. J.; Weston, M. H.; Sarjeant, A. A.; Nguyen, S. T.; Stair, P. C.; Snurr, R. Q. Vapor-Phase Metalation by Atomic Layer Deposition in a Metal-Organic Framework. J. Am. Chem. Soc. 2013, 135, 1029410297.

(12) Furukawa, H.; Gándara, F.; Zhang, Y. B.; Jiang, J.; Queen, W. L.; Hudson, M. R.; Yaghi, O. M. Water Adsorption in Porous MetalOrganic Frameworks and Related Materials. J. Am. Chem. Soc. 2014, 136, 4369-4381.

(13) Feng, D.; Wang, K.; Su, J.; Liu, T. F.; Park, J.; Wei, Z.; Bosch, M.; Yakovenko, A.; Zou, X.; Zhou, H. C. A Highly Stable Zeotype Mesoporous Zirconium Metal-Organic Framework with Ultralarge Pores. Angew. Chemie. Int. Ed. 2015, 54, $149-154$.

(14) Lammert, M.; Wharmby, M. T.; Smolders, S.; Bueken, B.; Lieb, A.; Lomachenko, K. A.; De Vos, D.; Stock, N. Cerium-Based Metal Organic Frameworks with UiO-66 Architecture: Synthesis, Properties and Redox Catalytic Activity. Chem. Commun. 2015, 51, 1257812581.

(15) Lammert, M.; Glißmann, C.; Reinsch, H.; Stock, N. Synthesis and Characterization of New Ce(IV)-MOFs Exhibiting Various Framework Topologies. Cryst. Growth. Des. 2017, 17, 1125-1131.

(16) Jacobsen, J.; Achenbach, B.; Reinsch, H.; Smolders, S.; Lange, F.-D.; Friedrichs, G.; De Vos, D.; Stock, N. The First Water-Based Synthesis of Ce(Iv)-MOFs with Saturated Chiral and Achiral C4-Dicarboxylate Linkers. Dalt. Trans. 2019, 48, 8433-8441.

(17) D'Amato, R.; Donnadio, A.; Carta, M.; Sangregorio, C.; Tiana, D.; Vivani, R.; Taddei, M.; Costantino, F. Water-Based Synthesis and Enhanced $\mathrm{CO}_{2}$ Capture Performance of Perfluorinated Cerium-Based Metal-Organic Frameworks with UiO-66 and MIL-140 Topology. ACS Sustain. Chem. Eng. 2019, 7, 394-402. 
(18) Nouar, F.; Breeze, M. I.; Campo, B. C.; Vimont, A.; Clet, G.; Daturi, M.; Devic, T.; Walton, R. I.; Serre, C. Tuning the Properties of the UiO-66 Metal Organic Framework by Ce Substitution. Chem. Commun. 2015, 51, 14458-14461.

(19) Waitschat; S.; Fröhlich,D.; Reinsch, H.; Terraschke, H.; Lomachenko, K. A.; Lamberti, C.; Kummer, H.; Helling, T.; Baumgartner, M.; Henninger, S.; Stock, N. Synthesis of M-UiO-66 (M = Zr, Ce or Hf)Employing 2,5-pyridinedicarboxylic acid as a linker: Defect Chemistry, Framework Hydrophilisation and Sorption Properties. Dalton Trans 2018, 47, 1062-1070.

(20) Silva, C. G.; Corma, A.; García, H. Metal-Organic Frameworks as Semiconductors. J. Mater. Chem. 2010, 20, 3141-3156.

(21) Zhan, W., Sun, L.; Han, X. Recent Progress on Engineering Highly Efficient Porous Semiconductor Photocatalysts Derived from MetalOrganic Frameworks. Nano-Micro Lett. 2019, 11, 1-28.

(22) Dhakshinamoorthy, A.; Li, Z.; Garcia, H. Catalysis and Photocatalysis by Metal Organic Frameworks. Chem. Soc. Rev. 2018, 81348172.

(23) Silva, C. G.; Luz, I.; Llabrés I Xamena, F. X.; Corma, A.; García, H. Water Stable Zr-Benzenedicarboxylate Metal-Organic Frameworks as Photocatalysts for Hydrogen Generation. Chem. - A Eur. J. 2010, 16, 11133-11138.

(24) Shen, L.; Liang, S.; Wu, W.; Liang, R.; Wu, L. Multifunctional $\mathrm{NH}_{2}$-Mediated Zirconium Metal-Organic Framework as an Efficient Visible-Light-Driven Photocatalyst for Selective Oxidation of Alcohols and Reduction of Aqueous Cr(Vi). Dalt. Trans. 2013, 42, 1364913657.

(25) Goh, T. W.; Xiao, C.; Maligal-Ganesh, R. V.; Li, X.; Huang, W. Utilizing mixed-linker zirconium basedmetal-organic frameworks to enhance the visible light photocatalytic oxidation of alcohol. Chem. Eng. Sci. 2015, 124, 45-51.

(26) Wu, X. P.; Gagliardi, L.; Truhlar, D. G. Cerium Metal-Organic Framework for Photocatalysis. J. Am. Chem. Soc. 2018, 140, 7904-7912.

(27) Julien, P.A.; Mottillo, C.; Friščić, T. Metal-organic Frameworks Meet Scalable and Sustainable Synthesis. Green Chem. 2017, 19, 27292747.

(28) Užarević, K.; Wang, T.C.; Moon, S.-Y.; Fidelli, A.M.; Hupp, J.T.; Farha, O.K.; Friščić, T. Mechanochemical and Solvent-Free Assembly of Zirconium Based Metal-organic Frameworks. Chem. Comm. 2016, 52, 2133-2136.

(29) He, Q.; Liu, J.; Li, Z.; Li, Q.; Xu, L.; Zhang B.; Meng, J.; Wu, Y.; Mai, L. Solvent-free Synthesis of Uniform MOF Shell-Derived Carbon Confined SnO2/Co Nanocubes for Highly Reversible Lithium Storage. Small, 2017, 13, 1701504-

(30) Beldon, P.J.; Fábián, L.; Stein, R.S.; Thirumurugan, A.; Cheetham, A.K.; Friščić, T., Rapid Room- Temperature Synthesis of Zeolitic Imidazolate Frameworks by Using Mechanochemistry. Angew. Chem. Int. Ed. 2010, 49, 9640-9643.

(31) James, S.L.; Adams, C.J., Bolm, C.; Braga, D.; Collier, P.; Friččić, T.; Grepioni, F.; Harris, K.D.M.; Hyett, G.; Jones, W.; Krebs, A.; Mack, J.; Maini, L.; Orpen, A.G.; Parkin, I.P.; Shearouse, W.C.; Steed, J.W.; Waddell, D.C. Mechanochemistry: opportunities for new and cleaner synthesis. Chem. Soc. Rev., 2012, 41, 413-447.

(32) Julien, P.A.; Užarević, K.; Katsenis, A.D.; Kimber, S.A. J.; Wang, T.; Farha, O.K.; Zhang, Y.; Casaban, J.; Germann, L.S.; Etter, M.; Dinnebier, R.E.; James, S.L.; Halasz, I.; Friščić, T. In situ Monitoring and Mechanism of the Mechanochemical Formation of a Microporous MOF-74 Framework. J. Am. Chem. Soc., 2016, 138, 2929-2932.

(33) Sanchez-Sala, M.; Vallcorba, O.; Domingo, C.; Ayllón, J. A. Acetic Acid as a Solvent for the Synthesis of Metal-Organic Frameworks Based on Trimesic Acid. Polyhedron 2019, 170, 458-462.

(34) D'Amato, R.; Marmottini, F.; McPherson, M.; Taddei, M.; Costantino, F. A Green, Solvent-Free Route to Functionalised Metal-Organic Frameworks with UiO-66 Topology. 2019, https://doi.org/10.26434/chemrxiv.8966105.

(35) Waitschat, S.; Fröhlich, D.; Reinsch, H.; Terraschke, H.; Lomachenko, K. A.; Lamberti, C.; Kummer, H.; Helling, T.; Baumgartner, M.; Henninger, S.; Stock, N. Synthesis of M-UiO-66 (M = Zr, Ce or Hf) Employing 2,5-Pyridinedicarboxylic Acid as a Linker: Defect Chemistry, Framework Hydrophilisation and Sorption Properties. Dalton. Trans. 2018, 47, 1062-1070.

(36) Guillerm, V.; Ragon, F.; Dan-Hardi, M.; Devic, T.; Vishnuvarthan, M.; Campo, B.; Vimont, A.; Clet, G.; Yang, Q.; Maurin, G.; Ferey, G.; Vittadini, A.; Gross, S.; Serre, C. A series of isoreticular, highly stable, porous zirconium oxide based metal-organic frameworks. Angew. Chem. Int. Ed. 2012, 51, 9267-9271.

(37) Baciocchi, E.; Del Giacco, T.; Rol, C.; Sebastiani, G. V. Cerium(IV) Ammonium Nitrate Catalyzed Photochemical Autoxidation of Alkylbenzenes. Tetrahedron Letters 1985, 26, 3353-3356.

(38) Higashimoto, S.; Kitao, N.; Yoshida, N.; Sakura, T.; Azuma, M.; Ohue, H.; Sakata, Y. Selective Photocatalytic Oxidation of Benzyl Alcohol and Its Derivatives into Corresponding Aldehydes by Molecular Oxygen on Titanium Dioxide under Visible Light Irradiation. J. Catal. 2009, 266, 279-285.

(39) Marotta, R.; Di Somma, I.; Spasiano, D.; Andreozzi, R.; Caprio, V. Selective Oxidation of Benzyl Alcohol to Benzaldehyde in Water by $\mathrm{TiO}_{2} / \mathrm{Cu}(\mathrm{II}) / \mathrm{UV}$ Solar System. Chem. Eng. J. 2011, 172, 243-249.

(40) Zhao, S.-N.; Wang, G.; Poelman, D.; Van Der Voort, P. Metal Organic Frameworks Based Materials for Heterogeneous Photocatalysis. Molecules 2018, 23, 2947-2970.

(41) Lang, X.; Chen, X.; Zhao, J. Heterogeneous Visible Light Photocatalysis for Selective Organic Transformations. Chem. Soc. Rev. 2014, 43, 473-486.

(42) Amori, L.; Del Giacco, T.; Rol, C.; Sebastiani, G. V. Photo-oxidation of Benzyl Alcohols Sensitized by $\mathrm{TiO}_{2}$ in $\mathrm{CH}_{3} \mathrm{CN}_{\text {in }}$ the Presence of $\mathrm{Ag}_{2} \mathrm{SO}_{4}$. Kinetic Evidence for the Involvement of Adsorption Phenomena. J. Chem. Res. (S) 1998, 10, 644-645.

(43) Del Giacco, T.; Faltoni, A.; Elisei, F. Anomalous Reactivity of Radical Cations Produced by Photosensitized Oxidation of 4-Methoxybenzyl Alcohol Derivatives: Role of the Sensitizer. Phys. Chem. Chem. Phys. 2008, 10, 200-210.

(44) Kresse, G.; Hafner, J. Ab Initio Molecular-Dynamics Simulation of the Liquid-Metal-Amorphous-Semiconductor Transition in Germanium. Phys. Rev. B 1994, 49, 14251-14269.

(45) Kresse, G.; Furthmüller, J. Efficiency of Ab-Initio Total Energy Calculations for Metals and Semiconductors Using a Plane-Wave Basis Set. Comput. Mater. Sci. 1996, 6, 15-50. 
(46) Hendrickx, K.; Vanpoucke, D. E. P.; Leus, K.; Lejaeghere, K.; Van Yperen-De Deyne, A.; Van Speybroeck, V.; Van Der Voort, P.; Hemelsoet, K. Understanding Intrinsic Light Absorption Properties of UiO-66 Frameworks: A Combined Theoretical and Experimental Study. Inorg. Chem. 2015, 54, 10701-10710.

Table of Content Graphics

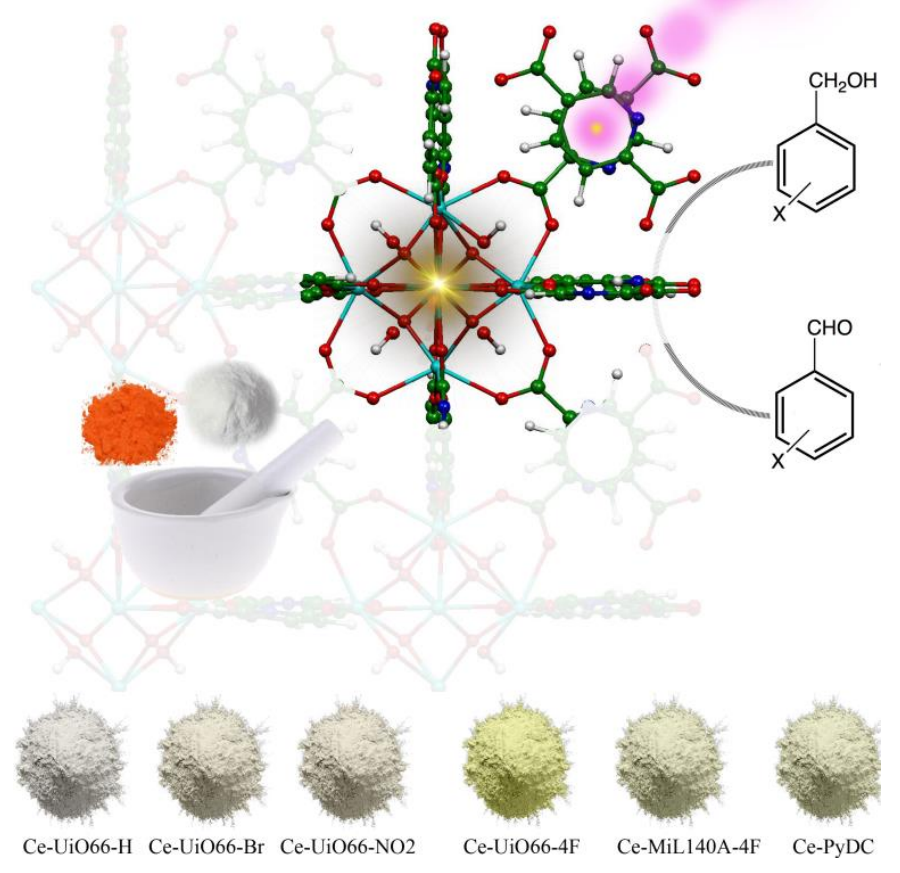

